\title{
GENERATORS OF BROWNIAN MOTIONS ON ABSTRACT WIENER SPACES
}

\author{
KEI HARADA \\ Graduate School of Mathematics, Nagoya University, Nagoya 464-8602, Japan \\ E-mail: kei.harada@math.nagoya-u.ac.jp
}

\begin{abstract}
We prove that Brownian motion on an abstract Wiener space $B$ generates a locally equicontinuous semigroup on $C_{b}(B)$ equipped with the $T_{t}$-topology introduced by L. Le Cam. Hence we obtain a "Laplace operator" as its infinitesimal generator. Using this Laplacian, we discuss Poisson's equation and heat equation, and study its properties, especially the difference from the Gross Laplacian.
\end{abstract}

1. Introduction. The problem to formulate the calculus on infinite dimensional spaces has attracted many mathematicians over one century. Even if we restrict our attention to Laplace operators, we can find many researches. In earlier times, many studied the theory of mean values on infinite dimensional spaces instead of Lebesgue measures. In this context, P. Lévy constructed his Laplacian, the so called Lévy Laplacian. This is different from the one defined by V. Volterra (see [11, 7] for instance). Later, L. Gross formulated his Laplacian in 2], the so called Gross Laplacian. He defined it as the trace of the second Fréchet derivative. His Laplacian is a direct extension of that of finite dimensional spaces. If we regard the Gaussian measure as the background measure, the Dirichlet form and Laplace-Beltrami operator are defined successfully (see e.g. [10] for details).

In this paper, we define a Laplace operator on abstract Wiener spaces via semigroups. As is mentioned by Gross $([2,3])$, one natural way to define a Laplace operator is to set

$$
\frac{1}{2} \Delta f=\lim _{t \downarrow 0} \frac{P_{t} * f-f}{t}
$$

on some function space, where $P_{t}$ is the Wiener measure with variance parameter $t$.

When $X=\mathbb{R}^{n}$, this process is successfully done on $L^{2}\left(\mathbb{R}^{n}\right)$. However, as is well known, the Lebesgue measure does not exist unless the space $X$ is locally compact, which is almost equivalent to assuming $X \subset \mathbb{R}^{n}$. Hence, the $L^{2}$ wonderland is not available when $X$ is

2000 Mathematics Subject Classification: 47D06, 60J45.

Key words and phrases: Gross Laplacian, $T_{t}$-topology, heat equations in infinite dimensions. The paper is in final form and no version of it will be published elsewhere. 
infinite dimensional. This problem leaves us a fundamental question, "On which function space should we calculate?"

Our choice in this paper is $\left(C_{b}(X), T_{t}\right)$, the space of bounded continuous functions equipped with the topology $T_{t}$ introduced by L. Le Cam [9]. Needless to say, the space $C_{b}(X)$ exists on any topological space, and the continuity is prescribed by that on each compact sets in many cases (in the case that $X$ is a $k$-space). Furthermore, the $T_{t}$-topology is described by the measures on $X$, and measures have tightness in most cases (such as Polish spaces), this enables us, with the help of Prohorov's theorem, to calculate as if $X$ were locally compact or $\sigma$-compact.

In this function space we prove that the semigroup mentioned before (heat semigroup) is strongly continuous and locally equicontinuous, and define our Laplacian as its generator. Our Laplacian appears to be similar to, but different from the Gross Laplacian.

We follow the terminology of [1, 2]. In this paper, all positive and signed measures are assumed to be bounded. We will denote by $M(X)$ the space of signed Borel measures. A positive Borel measure $\mu$ is said to be tight or compact regular if

$$
\mu(C)=\sup \{\mu(K): K \text { compact, } K \subset C\}
$$

for any Borel set $C$. A signed Borel measure $\mu$ is said to be tight if its total variation measure $|\mu|$ is tight. The total variation norm is defined by $\|\mu\|=|\mu|(X)$. A subset $\mathcal{M} \subset M(X)$ is said to be uniformly tight if it is bounded in the norm and there exist compact sets $\left\{K_{n}\right\}$ satisfying

$$
|\mu|\left(X \backslash K_{n}\right)<\frac{1}{2^{n}}
$$

for all $\mu \in \mathcal{M}$ and $n \in \mathbb{N}$.

We denote by $\tau_{w}$ the weak* topology $\sigma\left(M(X), C_{b}(X)\right)$ on $M(X)$. A topological space $X$ is called a Radon space if every signed Borel measure on $X$ is tight. $X$ is said to be a Prohorov space if any relatively compact subset in $\tau_{w}$ is uniformly tight. A Prohorov space is a Radon space. It is well known that any Polish metric space is a Prohorov space. $X$ is called a $k$-space if a set is closed whenever its intersection with each compact set is closed. Any metric or locally compact Hausdorff space is a $k$-space.

2. The $T_{t}$-topology. In this section we will be concerned with the $T_{t}$-topology on $C_{b}(X)$. This topology was first introduced by L. Le Cam 9 .

Definition 2.1. Let $\left\{K_{n}\right\}$ denote an increasing sequence of compact sets in $X$. For each $\left\{K_{n}\right\}$, let

$$
M_{\left\{K_{n}\right\}}=\left\{\mu \in M(X):\|\mu\| \leq 1,|\mu|\left(X \backslash K_{n}\right) \leq 1 / 2^{n}\right\}
$$

and set a seminorm $|\cdot|_{\left\{K_{n}\right\}}$ on $C_{b}(X)$ by

$$
|f|_{\left\{K_{n}\right\}}=\sup \left\{|\langle f, \mu\rangle|: \mu \in M_{\left\{K_{n}\right\}}\right\},
$$

where $\langle f, \mu\rangle$ denotes the integration. $T_{t}$ on $C_{b}(X)$ is the topology generated by the collection of these seminorms.

In other words, $T_{t}$ is the topology of uniform convergence on uniformly tight measures. Let $T_{K}$ denote the topology of uniform convergence on compact sets, and let $T_{u}$ denote that of uniform convergence on $X$, then $T_{K} \leq T_{t} \leq T_{u}$. Assume $M \subset C_{b}(X)$ is bounded 
in the norm, then $T_{t}$ and $T_{K}$ coincide on $M$. It is known that if $X$ is a $k$-space, then $\left(C_{b}(X), T_{t}\right)$ is complete. If $X$ is a Radon space, then the dual of $\left(C_{b}(X), T_{t}\right)$ is $M(X)$. If $X$ is a Prohorov space, then $T_{t}$ coincides with the Mackey topology $\tau\left(C_{b}(X), M(X)\right)$. See [1, 9] for details.

Compared with the norm topology $T_{u}, T_{t}$ might seem unnatural. However, the dual of $\left(C_{b}(X), T_{t}\right)$ is measures, while the dual of $\left(C_{b}(X), T_{u}\right)$ is finitely additive measures. Furthermore, $\left(C_{b}(X), T_{t}\right)$ is something like a $C^{*}$-algebra, though it is not a Banach space, in the sense that the pointwise product is continuous (Theorem 2.3). To prove this, we first prove the following lemma.

LEMMA 2.2. $|\cdot|_{\left\{K_{n}\right\}}$ is equivalent with the seminorm

$$
|f|_{\left[K_{n}\right]}=\sum_{n=1}^{\infty} \frac{1}{2^{n}}|f|_{K_{n}}
$$

where we set $|f|_{K_{n}}=\sup _{x \in K_{n}}|f(x)|$.

Proof. For any $\mu \in M_{\left\{K_{n}\right\}}$ and $f \in C_{b}(X)$, we have

$$
\begin{aligned}
\left|\int_{X} f(x) d \mu(x)\right| \leq & \int_{K_{1}}|f(x)| d \mu(x)+\int_{K_{2} \backslash K_{1}}|f(x)| d \mu(x) \\
& +\cdots+\int_{K_{n} \backslash K_{n-1}}|f(x)| d \mu(x)+\int_{X \backslash K_{n}}|f(x)| d \mu(x) \\
\leq & \sum_{m=1}^{n} \frac{1}{2^{m-1}} \sup _{x \in K_{m}}|f(x)|+\frac{1}{2^{n}}\|f\|
\end{aligned}
$$

for all $n \in \mathbb{N}$. Hence we obtain $|f|_{\left\{K_{n}\right\}} \leq 2|f|_{\left[K_{n}\right]}$.

Now we prove the converse inequality. Given $f \in C_{b}(X)$, we can find a sequence $\left\{x_{n}\right\}$ such that $x_{n} \in K_{n}$ and $\sup _{x \in K_{n}}|f(x)|=\left|f\left(x_{n}\right)\right|$. Set

$$
\mu=\sum_{n=1}^{\infty} \frac{1}{2^{n}} \delta_{x_{n}}
$$

then $\mu \in M_{\left\{K_{n}\right\}}$. Hence we obtain $|f|_{\left\{K_{n}\right\}} \geq|f|_{\left[K_{n}\right]}$.

THEOREM 2.3. The pointwise product on $C_{b}(X)$ is continuous in the $T_{t}$-topology.

Proof. Let $\left\{K_{n}\right\}$ be given. Set $K_{n}^{\prime}=K_{3 n}$, then

$$
|f|_{K_{3 n+k}} \leq|f|_{K_{n+1}^{\prime}} \leq 2^{n}|f|_{\left[K_{n}^{\prime}\right]},
$$

where $k=0,1,2$. The same holds for $g$, hence we obtain

$$
|f g|_{\left[K_{n}\right]} \leq \sum_{n=1}^{\infty} \sum_{k=0}^{2} \frac{1}{2^{3 n+k}}|f g|_{K_{3 n+k}} \leq \sum_{n=1}^{\infty} \sum_{k=0}^{2} \frac{1}{2^{n+k}}|f|_{\left[K_{n}^{\prime}\right]}|g|_{\left[K_{n}^{\prime}\right]} \leq|f|_{\left[K_{n}^{\prime}\right]}|g|_{\left[K_{n}^{\prime}\right]}
$$

which shows the continuity.

\section{Laplace operators on abstract Wiener spaces}

3.1. Definition of Laplacian. Let $(H, B, i)$ be an abstract Wiener space [4]; $H$ is a real separable Hilbert space, $B$ is the completion of $H$ with respect to some measurable 
norm $\|\cdot\|$ (hence $B$ is a real separable Banach space), $i: H \rightarrow B$ is the natural injection. The measure $P_{t}$ on $B$ is the Wiener measure on $B$ with variance parameter $t>0$. We set $P_{0}=\delta_{0}$, the delta measure in origin.

Let $p_{t}$ denote the operator on $C_{b}(B)$ defined by

$$
\left(p_{t} f\right)(x)=\left(P_{t} * f\right)(x)=\int_{B} f(x-y) d P_{t}(y) .
$$

Obviously $p_{0}=I, p_{t} p_{s}=p_{t+s}$. This is the semigroup generated by the Brownian motion on $B$.

It is known that $\left\{p_{t}\right\}_{t \geq 0}$ form a strongly continuous semigroup on the Banach space of bounded uniformly continuous functions (see [2, Proposition 6]). However, this space is small for infinite dimensional analysis. In this section, we prove that $\left\{p_{t}\right\}_{t \geq 0}$ form a strongly continuous locally equicontinuous semigroup on $\left(C_{b}(B), T_{t}\right)$. As a result, we obtain the generator, denoted by $\frac{1}{2} \Delta$, which is a densely defined closed operator on $\left(C_{b}(B), T_{t}\right)$.

Proposition 3.1. For all $s>0,\left\{p_{t}\right\}_{0 \leq t \leq s}$ are equicontinuous linear operators.

Proof. Fix $\varepsilon>0$. Since a real separable Banach space $B$ is a Radon space, we have $P_{s}$ is tight. Hence there exists a compact set $K$ such that

$$
P_{s}(B \backslash K)<\varepsilon .
$$

Set $K^{\prime}=\{t x: x \in K, t \in[0,1]\}$, then $K^{\prime}$ is a compact set with $r K^{\prime} \subset K^{\prime}$ for all $0 \leq r \leq 1$. By the equation $p_{t s}(E)=p_{t}\left(s^{-1 / 2} E\right)$ (equation (3) in [2]), we have

$$
P_{t}\left(K^{\prime}\right) \geq P_{t}\left(\sqrt{t / s} K^{\prime}\right)=P_{s}\left(K^{\prime}\right) \geq P_{s}(K) \geq 1-\varepsilon .
$$

Therefore, the measures $\left\{P_{t}: 0 \leq t \leq s\right\}$ are uniformly tight. Since

$$
\left\langle p_{t} f, \mu\right\rangle=\left\langle P_{t} * f, \mu\right\rangle=\left\langle f, P_{t} * \mu\right\rangle
$$

for any $\mu \in M(B)$ and $f \in C_{b}(B)$, it follows that

$$
\left|p_{t} f\right|_{\left\{K_{n}\right\}} \leq|f|_{\left\{K_{n}^{\prime}\right\}}
$$

for some $\left\{K_{n}^{\prime}\right\}$ by the following lemma.

Lemma 3.2. Let $\mathcal{M}_{1}, \mathcal{M}_{2} \subset M(B)$ be uniformly tight, then the set

$$
\left\{\mu_{1} * \mu_{2}: \mu_{1} \in \mathcal{M}_{1}, \mu_{2} \in \mathcal{M}_{2}\right\}
$$

is uniformly tight.

Proof. For any $\varepsilon>0$, there exist compact sets $K_{1}, K_{2}$ such that $\left|\mu_{1}\right|\left(B \backslash K_{1}\right)<\varepsilon$ for all $\mu_{1} \in \mathcal{M}_{1}$ and $\left|\mu_{2}\right|\left(B \backslash K_{2}\right)<\varepsilon$ for all $\mu_{2} \in \mathcal{M}_{2}$. Set

$$
K_{3}=\left\{x_{1}+x_{2}: x_{1} \in K_{1}, x_{2} \in K_{2}\right\} .
$$

$K_{3}$ is a compact set satisfying

$$
\begin{aligned}
\left(\mu_{1} * \mu_{2}\right)\left(B \backslash K_{3}\right) \leq & \left(\mu_{1} \times \mu_{2}\right)\left(\left(B \backslash K_{1}\right) \times B\right) \\
& +\left(\mu_{1} \times \mu_{2}\right)\left(B \times\left(B \backslash K_{2}\right)\right) \\
\leq & \left(\left\|\mu_{1}\right\|+\left\|\mu_{2}\right\|\right) \varepsilon
\end{aligned}
$$

where $\mu_{1} \times \mu_{2}$ is the direct product. 
It remains to prove that the map $t \mapsto p_{t} f$ is strongly continuous.

LEMMA 3.3. Fix $f \in C_{b}(B), s>0$, and a compact set $K$, then for any $\varepsilon>0$, there exists $\delta>0$ such that: $x, y \in K$ and $\|x-y\|<\delta \Rightarrow\left|p_{t} f(x)-p_{t} f(y)\right|<\varepsilon$ for any $0 \leq t \leq s$.

Proof. Since $\left\{P_{t}\right\}_{0 \leq t \leq s}$ is uniformly tight, there exists a compact set $K^{\prime}$ with the property that

$$
P_{t}\left(K^{\prime}\right)>1-\frac{\varepsilon}{4\|f\|}
$$

for all $0 \leq t \leq s$. Set

$$
K^{\prime \prime}=\left\{x+x^{\prime}: x \in K, x^{\prime} \in K^{\prime}\right\} .
$$

As $K^{\prime \prime}$ is compact, we can choose $\delta>0$ so that $x, y \in K^{\prime \prime}$ and $\|x-y\|<\delta \Rightarrow \mid f(x)-$ $f(y) \mid<\varepsilon / 2$.

Now we obtain

$$
\begin{aligned}
\left|p_{t} f(x)-p_{t} f(y)\right| \leq & \int|f(x-z)-f(y-z)| d P_{t}(z) \\
& =\int_{K^{\prime \prime}}|f(x-z)-f(y-z)| d P_{t}(z) \\
& +\int_{\left(K^{\prime \prime}\right)^{c}}|f(x-z)-f(y-z)| d P_{t}(z) \\
& <\frac{\varepsilon}{2}+2\|f\| \frac{\varepsilon}{4\|f\|}=\varepsilon .
\end{aligned}
$$

LEMma 3.4. For any $\varepsilon>0$ and $\delta>0$, there exists $t_{0}>0$ such that;

$$
P_{t}\left(\overline{B_{0}(\delta)}\right)>1-\varepsilon
$$

for all $0 \leq t \leq t_{0}$, where $B_{0}(\delta)$ denotes the open ball $\{x:\|x-0\|<r\}$, and the overline stands for the closure.

Proof. This follows immediately from the equation (3) in [2] and the fact that any compact set is bounded.

Proposition 3.5. For any $f \in C_{b}(B)$, the map $t \mapsto p_{t} f$ is continuous in $T_{t}$-topology.

Proof. Since $\left\{p_{t} f\right\}_{t \geq 0}$ is uniformly bounded, we only need to show the continuity in the topology $T_{K}$. Fix $\varepsilon>0$ and $t_{0}>0$. Let $K^{\prime}$ be a compact set satisfying

$$
P_{t}\left(K^{\prime}\right)>1-\varepsilon
$$

for all $0 \leq t \leq t_{0}$, and set

$$
K^{\prime \prime}=\left\{x+x^{\prime}: x \in K, x^{\prime} \in K^{\prime}\right\} .
$$

By Lemma 3.3 , there exists $\delta>0$ such that $x, y \in K^{\prime \prime}$ and $\|x-y\|<\delta \Rightarrow \mid p_{t} f(x)-$ $p_{t} f(y) \mid<\varepsilon$.

By Lemma 3.4, we have, for $0 \leq s<t \leq t_{0}$ with $s-t$ sufficiently small,

$$
P_{s-t}\left(K^{\prime \prime} \cap \overline{B_{0}(\delta)}\right)>1-\varepsilon .
$$


Under the conditions stated above, for all $x \in K$, we have

$$
\begin{aligned}
\left|p_{s} f(x)-p_{t} f(x)\right| \leq & \int_{B}\left|\left(p_{t} f(x-y)-p_{t} f(x)\right)\right| d P_{s-t}(y) \\
\leq & \int_{K^{\prime \prime} \cap \overline{B_{0}(\delta)}}\left|p_{t} f(x-y)-p_{t} f(x)\right| d P_{s-t}(y) \\
& +\int_{\left(K^{\prime \prime} \cap \overline{B_{0}(\delta)}\right)^{c}}\left|p_{t} f(x-y)-p_{t} f(x)\right| d P_{s-t}(y) \\
\leq & \varepsilon+2\|f\| \varepsilon,
\end{aligned}
$$

which shows the continuity.

Hence, by the Hille-Yosida theorem, we obtain the following theorem.

THEOREM 3.6. Let us denote by $\frac{1}{2} \Delta$ the infinitesimal generator of $\left\{p_{t}\right\}$. This is a densely defined closed operator on $\left(C_{b}(B), T_{t}\right)$.

3.2. Properties of Laplacian. Our Laplacian is an extension of the usual finite dimensional Laplacian.

Proposition 3.7. Let $e_{1}, \cdots, e_{n} \in B^{*}$ be orthonormal as elements of $H^{*}$, and let $g_{n}$ : $\mathbb{R}^{n} \rightarrow \mathbb{R}$ be $C_{b}^{2}$ functions, then $g(x)=g_{n}\left(p_{n}(x)\right) \in D(\Delta)$ and $\Delta g(x)=\Delta g_{n}\left(p_{n}(x)\right)$, where $p_{n}(x)=\left(\left\langle e_{1}, x\right\rangle, \cdots,\left\langle e_{n}, x\right\rangle\right)$.

Proof. Since

$$
\left(P_{t} * g\right)(x)=\left(P_{t}^{n} * g_{n}\right)\left(p_{n}(x)\right),
$$

where $P_{t}^{n}$ is the Gaussian measure on $\mathbb{R}^{n}$ with variance $t$,

$$
\lim _{t \rightarrow 0} \frac{\left(P_{t} * g\right)(x)-g(x)}{t}=\frac{1}{2} \Delta g_{n}\left(p_{n}(x)\right) \quad \text { in } T_{u} .
$$

Even though $p_{t} f$ is defined by "global" calculation, this Laplacian has "locality" in the following sense.

Proposition 3.8. Let $f \in D(\Delta)$, then supp $\Delta f \subset \operatorname{supp} f$.

Proof. It suffices to show $\Delta f(x)=0$ for $x \notin \operatorname{supp} f$. Since there exists $\delta>0$ such that $f=0$ on $B_{x}(\delta)$, by [2, Remark 2.3.],

$$
p_{t} f(x)=\int_{B_{x}(\delta)^{c}} f(x-y) d P_{t}(y) \leq\|f\| o(t) .
$$

Hence $\left(p_{t} f(x)-f(x)\right) / t \rightarrow 0$ as $t \rightarrow 0$.

REMARK 3.9. We used the fact $f$ is bounded, which is, in a way, global information.

3.3. Potentials. Now we will study the Poisson's equation

$$
\frac{1}{2} \Delta u=-f
$$

for given $f \in C_{b}(B)$. (Problems such as the Dirichlet problem will be studied in [6]) Following Gross, we assume $\operatorname{supp} f$ has the following property ([2, Remark 3.5]): for some $e_{1}, e_{2}, e_{3} \in B^{*}$ which are orthonormal as elements of $H^{*}$, each $e_{j}(j=1,2,3)$ is bounded on $\operatorname{supp} f$, that is, $\left|\left\langle e_{j}, x\right\rangle\right| \leq \exists M$ for all $x \in \operatorname{supp} f$. Note that we have not assumed that 
supp $f$ is bounded (which often means $f=0$ in infinite dimensional spaces). Of course, the solution is not unique (add constant).

THEOREM 3.10. The Poisson's equation has a solution under the condition stated above. Proof. Set $u_{s} \in C_{b}(B)$ by

$$
u_{s}=\int_{0}^{s} p_{t} f d t,
$$

for $s>0$. Obviously, $u_{s} \in D(\Delta)$ and

$$
\frac{1}{2} \Delta u_{s}=p_{s} f-f \text {. }
$$

Furthermore, by the assumption on $\operatorname{supp} f$, it follows that

$$
\left\|p_{T} f\right\| \leq t^{-3 / 2} C
$$

for some $C>0$, hence $p_{T} f \rightarrow 0$ and $u_{T} \rightarrow u$ uniformly as $T \rightarrow \infty$. Since $\Delta$ is closed, $u$ is a solution.

REMARK 3.11. This theorem shows our Laplacian is different form the Gross Laplacian, the trace of the second Fréchet derivative. Gross [2, Theorem 2] showed that there exists a bounded uniformly continuous function $f$ on $B$ with bounded support which is zero in the neighborhood of the origin and such that the second Fréchet derivative of $u$, defined in the same way as above, is not trace class at the origin. This shows that trace class is too strict a condition to deal with potentials. As Gross mentioned [2, Remark 4.4], there is some underlying summability method in this generator.

REMARK 3.12. Here we used the generator defined on $\left(C_{b}(B), T_{t}\right)$ as Laplacian. The same proof is possible for the generator defined on the Banach space of bounded uniformly continuous functions.

REMARK 3.13. The regularity of the potential is a difficult problem. The potential is something like $C^{1}$ [2, Lemma 2.1], but it is not true that the potential is something like $C^{2}$ in general, because it is not true even in the case of $R^{2}$.

3.4. Heat equations. Since we defined the Laplacian as the generator of the heat semigroup, it is natural to think that $u(t, x)=p_{t} f(x)$ is the solution of the following equation (initial value problem of heat equation):

$$
\begin{aligned}
\frac{\partial}{\partial t} u(t, x) & =\frac{1}{2} \Delta u(t, x), \\
u(0, x) & =f(x) .
\end{aligned}
$$

However, unlike in finite dimensional cases, it does not directly follow that $p_{t} f \in D(\Delta)$. This is because $\Delta P_{t}$ is not a bounded measure.

THEOREM 3.14. The map $f \mapsto \Delta p_{t} f$ defined for $f \in D(\Delta)$ cannot be extended to a continuous operator on $\left(C_{b}(B), T_{t}\right)$.

Proof. Assume there exists such an extension, then there exists a bounded measure $\mu$ such that

$$
\int f(x) d \mu(x)=\frac{1}{2}\left(p_{t} \Delta f\right)(0) .
$$


for $f \in D(\Delta)$. By Proposition 3.7 .

$$
\int g_{n}\left(p_{n}(x)\right) d \mu(x)=\int g_{n}(y) d\left(\frac{1}{2} \Delta P_{t}^{n}\right)(y)
$$

for $g_{n} \in C_{b}^{2}\left(\mathbb{R}^{n}\right)$. Since $\left\|\Delta P_{t}^{n}\right\| \rightarrow \infty$ as $n \rightarrow \infty, \mu$ is not a bounded measure.

REMARK 3.15. A similar result is obtained [5] in the settings of heat semigroups in Hilbert spaces (see e.g. [13]).

Regularity of the heat equation is interesting. For example, if it is analytic in some sense, it would give an approach to the Feynman's path integral. However, the theorem above means that the heat semigroup is not so much regular, if we are working on $\left(C_{b}(B), T_{t}\right)$. It seems that we need to use the regularity of initial condition $f$, or to find a better topology such that the unbounded measure $\Delta P_{t}$ is something like dual.

REMARK 3.16. If $f \in D(\Delta)$, then it is easy to see $u$ is the solution. Gross [2, Theorem 3] proved that if $f$ is Lip 1, then $(\partial u / \partial t)$ exists in the sense of uniform convergence. Hence $p_{t} f \in D(\Delta)$ in this case. The author is not sure whether $p_{t} f \in D(\Delta)$ for all $f \in C_{b}(B)$ and $t>0$. In other words, whether the continuity of $f$ (that is a regularity) is enough or not.

\section{References}

[1] D. H. Fremlin, D. J. H. Garling, and R. G. Haydon, Bounded measures on topological spaces, Proc. London Math. Soc. 25 (1972), 115-136.

[2] L. Gross, Potential theory on Hilbert space, J. Funct. Anal. 1 (1967), 123-181.

[3] L. Gross, Abstract Wiener measure and infinite dimensional potential theory, in: Lectures in Modern Analysis and Applications II, Lecture Notes in Math. 140, Springer, 1970, $84-116$.

[4] L. Gross, Abstract Wiener spaces, in: Proceedings of the Fifth Berkeley Symposium on Mathematical Statistics and Probability, Vol. II, Univ. of California Press, Berkeley, CA, 1967.

[5] P. Guiotto, Non-differentiability of heat semigroups in infinite dimensional Hilbert spaces, Semigroup Forum 55 (1997), 232-236.

[6] K. Harada, Laplace equations on abstract Wiener spaces, in preparation.

[7] T. Hida, H. H. Kuo, J. Potthoff, and L. Streit, White Noise, Kluwer, 1993.

[8] J. L. Kelley, General Topology, Springer, 1991.

[9] L. Le Cam, Convergence in distribution of stochastic processes, Univ. Calif. Publ. Statist. 2 (1957), 207-236.

[10] P. Malliavin, Stochastic Analysis, Springer, 1997.

[11] P. Lévy, Problèmes Concrets d'Analyse Fonctionnelle, Gauthier-Villars, Paris, 1951.

[12] K. R. Parthasarathy, Probability Measures on Metric Spaces, Academic Press, New York, 1967.

[13] J. Zabczyk, Parabolic equations on Hilbert spaces, in: Stochastic PDE's and Kolmogorov Equations in Infinite Dimensions, Lecture Notes in Math. 1715, Springer, 1998, 117-213. 